\title{
Análisis comparativo de la vegetación vascular asociada con los complejos cenagosos en la llanura aluvial del medio y bajo Atrato, Chocó Biogeográfico
}

\section{Comparative analysis of the vascular vegetation associated of wetlands complexes alluvial plain middle and low Atrato, Chocó Biogeographic}

\section{Zulmary Valoyes-Cardozo¹, Giovanny Ramírez-Moreno²}

\begin{abstract}
Resumen
En las últimas décadas, la vegetación que circunda los complejos cenagosos de la llanura aluvial del Atrato ha sido transformada debido a la explotación intensiva a la que ha sido sometida. El grado de afectación varía de acuerdo con la ubicación y con la intensidad del disturbio, situación que a su vez ha provocado el cambio en la composición y estructura de este importante recurso, cuyas consecuencias ecológicas son dificiles de valorar. En este sentido este artículo hace un análisis del estado actual de la vegetación que circunda estos ecosistemas y las implicaciones que ha tenido la transformación de la misma. Para lo anterior se tomó como base estudios realizados en los complejos cenagosos y ciénagas de la zona media y baja del Atrato y se comparó la vegetación presente en estos, concluyendo que aunque existen vacios de información referentes a la composición y estructura en la mayoría de estos complejos cenagosos; el ritmo acelerado con que se transforman estos ambientes ponen en riesgo la diversidad contenida en ellos y por consiguiente la seguridad alimentaria de las poblaciones asentadas en esta parte del territorio.
\end{abstract}

Palabras clave: Complejos cenagosos del Chocó Biogeográfico, Estado de la vegetación, Estructura vegetal, Gremios ecológicos, Llanura aluvial del Atrato.

\begin{abstract}
TIn the last decades, the vegetation that surrounds the wetlands complexes of the alluvial plain of the Atrato has been transformed due to the intensive exploitation to which it has been submitted, the degree of affectation changes in agreement to the location and to the intensity of the disturbance, situation that in turn has provoked the change in the composition and structures of this important resource, whose ecological consequences are difficult to value. In this respect this article, it does an analysis of the current condition of the vegetation that surrounds these ecosystems and the implications that the transformation of the same one has had. For the previous thing one took as a base studies realized in the wetlands complexes and marshes of the zone it happens and fall of the Atrato, was compared the present vegetation in these. Concluding that though there exist emptinesses of information relating to the composition and structure in the majority of these wetlands complexes, the intensive pace with which these environments transform they put in risk the diversity contained in them and consequently the food safety of the populations seated in this part of the territory
\end{abstract}

1 Bióloga, Especialista en Administración de Recursos Naturales, Estudiante de Maestría en Ciencias Ambientales. Investigadora Contratista, Instituto de Investigaciones Ambientales del Pacífico (IIAP), Quibdó, Colombia.

e-mail:zulmaryvaloyes@gmail.com

2 Biólogo, MSc en Ciencias Biológicas, Candidato a PhD. Investigador principal Componente Ecosistémico, Instituto de Investigaciones Ambientales del Pacífico (IIAP), Quibdó, Colombia. e-mail: gramirezm@iiap.org.co

Recibido: 15 de enero de 2012 Aceptado: 10 de mayo de 2012 
Keywords: Alluvial plain of the Atrato, Estate of the vegetation, Ecological guilds, Vegetal estructure, Wetlands complexes.

\section{Introducción}

Los humedales muestran una enorme diversidad de acuerdo con su origen, temporalidad, localización geográûca, régimen acuático y químico; en este sentido los humedales del río Atrato por las características regionales presentan un gran potencial de desarrollo para las comunidades asentadas en su zona de influencia. Las características ecológicas del ambiente permiten que los bosques asociados con este tipo de ecosistemas sean diversos, pues reciben continuamente contribuciones de materiales que proporcionan los diferentes nutrientes que ayudan al sostenimiento de la estructura y función de estos sistemas (Ramírez y Valoyes, 2009). A pesar de lo anterior muchas de las especies que estos ambientes albergan se encuentran amenazadas o al borde de la extinción como consecuencia de la destrucción de sus hábitats y la explotación irracional a la que se ven sometidas (Blanco, 2012). Otras amenazas como la extracción de agua para usos mineros, la contaminación por vertido de residuos domiciliarios e industriales, la agricultura extensiva, la ganadería, los monocultivos y la explotación forestal atentan contra las funciones de estos ecosistemas poniéndolos en riesgo de desaparecer (COMANA, 2005). Las actividades mencionadas han permitido que en términos generales la vegetación asociada con los diferentes microambientes que integran los complejos cenagosos presentes en la llanura aluvial del río Atrato, haya sido transformada casi en su totalidad, conduciendo a los humedales asociados con un cambio generalizado de cobertura boscosa, lo que a su vez ha causado serios impactos a la biodiversidad de la región.

Actividades como la minería aluvial practicada de forma ilegal en algunos tributarios de la llanura aluvial del Atrato, ha venido ocasionando fuertes impactos en el suelo y los recursos biológicos que integran este recurso, impulsando la erosión y desprendimiento del mismo en las márgenes de estas fuentes y el arrastre de sólidos suspendidos afectando de forma permanente la vida acuática. CORPOURABA y CODECHOCO (2006) reportan que esta actividad se practica con mayor intensidad en algunos tributarios en la zona media del río Atrato; en época seca la minería se realiza en la margen oriental del mismo Atrato. Al mismo tiempo, la explotación forestal practicada por personas locales y empresas madereras, sin técnicas de aprovechamiento adecuadas y con la inclusión de colonos dentro del bosque, realizaron la apertura de una red de caminos y construyeron una red de canales de transporte para la extracción de especies maderables de alto valor biológico y comercial, provocando efectos trascendentales como pérdida del recurso, hábitat y alimento de la fauna local, alteración de la estructura y composición del bosque, pérdida de la capa orgánica del suelo, degradación de fuentes hídricas, desvío del cauce natural del río y la reducción de su caudal por el constante arrastre de sedimentos, la disminución de recursos hidrobiológicos y una serie de conflictos sociales. Aldana (2011) reporta una tasa de pérdida de cobertura vegetal de 358,6 ha/año, equivalente a $3,6 \mathrm{~km}^{2}$, en un área de $630 \mathrm{~km}^{2}$, , lo que da una tasa anual de deforestación de $0,5 \%$ anual para la zona que es alta respecto al promedio nacional. Lo anterior ha producido cambios en la estructura y composición de las asociaciones que conforman los diferentes ambientes de esta vasta región, debido a los requerimientos ecológicos de cada especie. Especies particulares como Prioria copaifera, elemento principal de los bosques de cativo que integran el paisaje de la parte baja de la llanura aluvial, tarda más de 130 años en alcanzar el diámetro comercial (Condit et al., 1995), pues adquiere la edad biológica reproductiva y silvicultural a los 40 y $60 \mathrm{~cm}$ de DAP (CONIF y PIZANO, 1996). La complejidad ecológica de este tipo de especies y el ritmo de deforestación a que son sometidos este tipo de boques hacen casi imposible la regeneración natural de estos ambientes y la resiliencia de las especies que lo integran.

Por otro lado la agricultura que ha venido incrementando su desarrollo en suelos de vocación forestal y de forma itinerante, es una de las principales causas de la continua apertura del bosque. Aunque en algunas zonas de la llanura aluvial esta actividad es de subsistencia y el bosque se puede regenerar después de algunos años de abandono, en otras localidades es una actividad comercial con extensas áreas cultivadas. La década de 1950 marcó el inicio de la maldición de la agroindustria en el Atrato, pues el gobierno otorgó hectáreas de tierra en la zona para la siembra de la palma aceitera Elais guianensis (Sánchez, 2006). Entre los años 1994 y2001 el cultivo de la palma aceitera aumentó de 153 a 3.834 ha en territorios colectivos de comunidades negras (Mingorance et al., 2004, Herrera y Primera, 2007). El reemplazo de la vegetación natural e implementación del monocultivo de palma aceitera produjo la disminución del caudal de 12 cuerpos de agua en las cuencas de los ríos Curvaradó y Jiguamiandó (quebrada Cristalina, la Nevera, la Pradera, la Madre, la Morroca, la Iguana, Ciénagas la Cristalina, el Guacuco y los caños el Cerrao, el Cojo y el Claro), situación que a su vez produjo la desecación definitiva de las ciénegas Andalucía, Alvarado, los Bartolos y los Cativos (Cuesta y Ramírez, 2009). Esto produjo el desequilibrio de estos ambientes, disminuyendo la diversidad de la región. La agricultura extensiva tuvo sus inicios en esta región después de la introducción de la palma aceitera; en la actualidad se han calculado unas 10.000 ha de cultivo de yuca, unas 4.000 ha de plátano tipo exportación en los ríos Curvaradó y Jiguamiandó (INCODER, 2012), reemplazando así una agri- 


\section{Bioetnia Volumen 9 No 2 (julio-diciembre), 2012}

cultura itineraria y de subsistencia por la siembra de monocultivos, cuyo sostenimiento ha causado graves problemas ambientales en esta zona del Atrato, sustituyendo el ecosistema natural por el de la palma y otras especies.

Sin duda, otra actividad impactante en los bosques de esta zona es la ganadería, causante de la degradación de extensas áreas de bosque en las selvas de la llanura aluvial. Se estima que entre 6.000 a 90.000 ha de bosque se han dedicado a la explotación extractiva, lo que incluye la introducción de pastos y la ganadería extensiva en localidades como Curvaradó, Jiguamiandó, Acandí, Ungía y Riosucio (Marín et al., 2004; CODESARROLLO, 2006; Carmona, 2010; INCODER, 2012 y la Alcaldía Municipal de Riosucio, 2013). Consecuentemente los incendios forestales producidos por los pobladores locales como una medida para eliminar el bosque con fines agrícolas y la cacería (hicotea, babilla), han provocado la eliminación de gran parte de la vegetación que bordea las ciénegas, afectando a poblaciones de interés biológico como los panganales de Raphia taedigera (eltiempo.com, 1992; PNN los Katíos, 2008; Chocó 7 días, 2011). El objetivo de este documento es considerar el estado de conservación de la flora vascular asociada con los complejos cenagosos de la llanura aluvial del Atrato, a partir de la revisión de información secundaria y de los análisis cualitativos obtenidos a través de datos de campo, tomando como variable la presencia ausencia de especies de alto valor biológico y comercial.

\section{Metodología}

Área de estudio. El río Atrato nace en los Altos de la Concordia y los Farallones de Citará en el cerro de Caramanta, entre los $5^{\circ}$ y $8^{\circ}$ de latitud norte y $\operatorname{los} 76^{\circ}$ y $78^{\circ}$ de longitud oeste, en el extremo noroccidental de Colombia y de Suramérica a una altura de $3900 \mathrm{msnm}$. La cuenca del Atrato se encuentra delimitada al este por la cordillera Occidental y al oeste por las serranías del Baudó y del Darién, al sur se encuentra la divisoria de aguas con el río San Juan definida por el istmo de Istmina. La desembocadura del río Atrato se encuentra en el Golfo de Urabá, sobre el mar Caribe, donde forma un complejo sistema deltaico (Figura 1). El Atrato presenta una longitud en la cuenca media de 180 km entre los municipios de Quibdó y Bellavista, una profundidad media de 11 metros y 282 metros de ancho en promedio. Elárea de drenaje es de 806.477 hectáreas, de las cuales 130.000 corresponden a llanura aluvial, por lo cual, es considerado como uno de los ríos más caudalosos del mundo. La cuenca del trato está dividida en tres grades zonas: Alto Atrato, Medio Atrato y Bajo Atrato (MinAmbiente et al., 2002; CODECHOCO y CORPOURABA, 2006).

La llanura aluvial del río Atrato está constituida por terrenos sujetos a inundaciones permanentes a lado y lado del Atrato; presenta una textura suava y homogénea, donde se integran ciénagas y terrenos cenagosos. Se encuentra en parches de diferentes colores, correspondiente a franjas de vegetación, a causa de disponibilidades hídricas y freáticas diversas. Los colores están en la gama del verde marrón, intercalados con parches oscuros que presentan los cuerpos de agua. Los efectos del río Atrato y sus afluentes río León y los niveles freáticos superficiales hacen que la vegetación dependa del régimen hídrico (Corporación OSSO, 1998).

El tramo final del río, presenta un extenso plano de inundación conformado por ciénagas y caños de concxión entre ellas y el cauce principal del río (Jaramillo y Jiménez, 2008). Estos ecosistemas constituyen el hábitat de muchas especies de flora y fauna. Las ciénagas, que en realidad son lagos pantanosos, son verdaderos santuarios de biodiversidad porque albergan una variada fauna y una vegetación característica de estos ambientes que incluye lechuga acuática, lirio acuático, el tabaquillo y otras característica de la zona como el arracacho que se comporta como nicho para el desarrollo de los peces (Hidalgo, 2003).

La ubicación de la cuenca del río Atrato en el extremo noroccidental de América del Sur, entre el litoral Pacífico y el Caribe, en la zona Ecuatorial, define las características climáticas de la región (Rodríguez, 2012). El clima es cálido húmedo, con alturas menores de $1000 \mathrm{msnm}$, precipitaciones de 2.000 a $4.000 \mathrm{~mm}$ y temperatura promedio anual mayor de $24^{\circ} \mathrm{C}$. De acuerdo con Holdridge, corresponde a la zona de vida de bosque húmedo tropical (bh-T). El relieve predominante es plano y ligeramente plano, con pendientes $0-1 \%$ y 1 $3 \%$. El material parental que da origen a los suelos evolucionó a partir de sedimentos aluviales, constituidos sobre todo por arcillas y limos. Los suelos mejor drenados están dedicados casi todos a los cultivos de subsistencia como arroz, yuca, plátano, caña de azúcar y frutales; las áreas que presentan drenaje pobre, por lo general tienen vegetación natural hidrófila, constituida esencialmente por palmas, ciperáceas y heliconias (INVIAS y IIAP, 2013).

Métodos. La información base para el análisis del estado de la vegetación asociada con los humedales que integran la zona media y baja de la llanura aluvial del Atrato, se obtuvo de diferentes estudios específicos desarrollados en estos ecosistemas en diferentes épocas (evaluación de las Ciénegas Tumarado, Perancho, la Honda, la Rica en el bajo Atrato, 2002) y las caracterizaciones ecológicas de los tres complejos cenagosos ubicados en el medio Atrato (C. Grande de Beté, 2008, C. la Larga, Tagachí, 2012 y C. la Honda, Tanguí, 2013), realizados por el Instituto de Investigaciones Ambientales del Pacífico (IIAP); de igual forma se contó con el plan de manejo de los humedales del medio y bajo Atrato, publicado por CODECHOCO y CORPOURABA (2006) y la caracterización de las ciénagas de Marriaga y Limón realizada por CODECHOCO y CORPOARIEN (2012) así como con información disponible en la web. 
Vegetación vascular y los complejos cenagosos. Z. Valoyes-Cardozo, G. Ramírez-Moreno

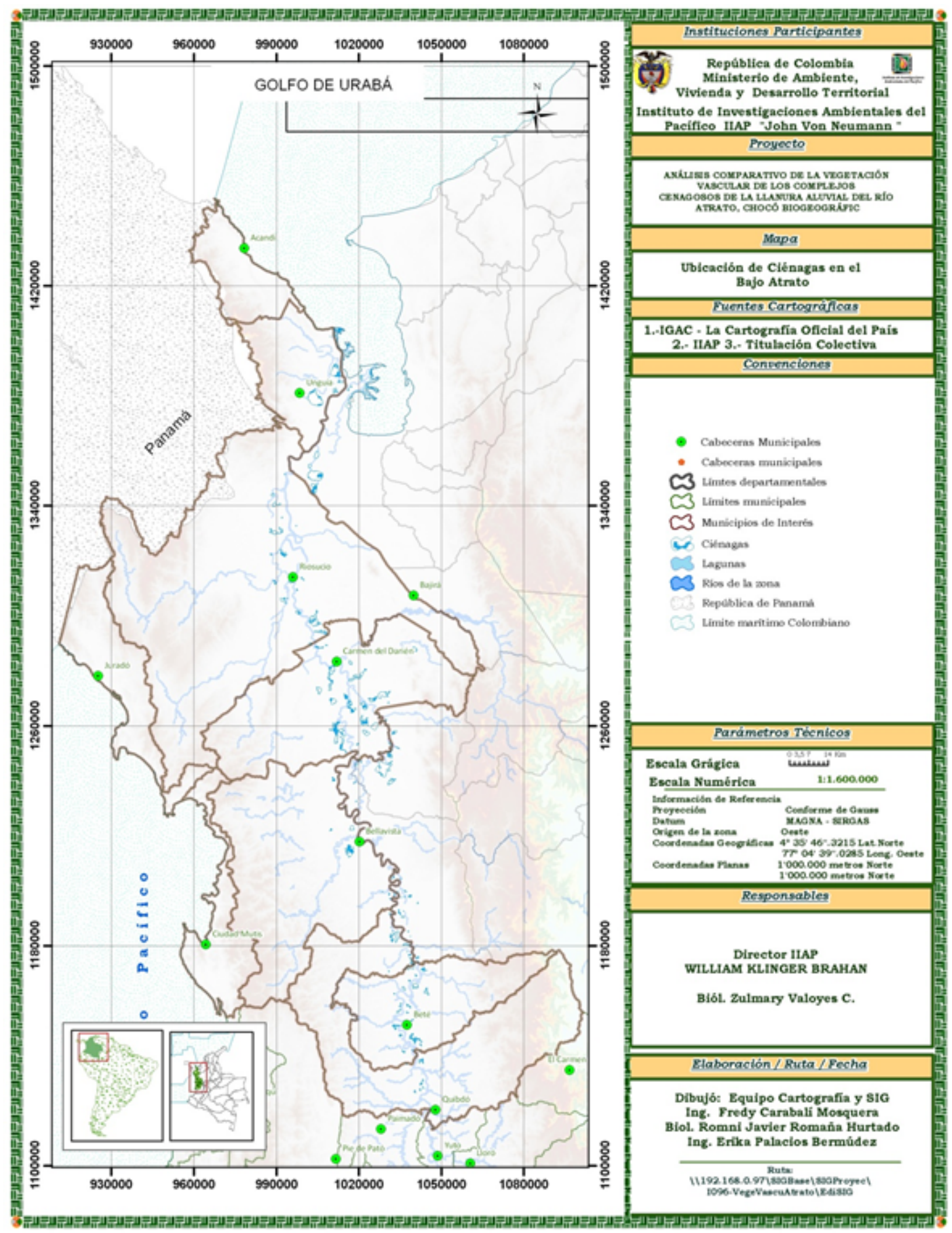

Figura 1. Ubicación de la llanura aluvial del río Atrato 


\section{Bioetnia Volumen 9 No 2 (julio-diciembre), 2012}

\section{Resultadosy discusión}

En la llanura aluvial del Atrato se calcularon unas 960 ha de humedales, correspondientes a bosque inundables y ciénagas (CODECHOCO y CORPOURABA, 2006), los últimos en su mayoría se conectan con la fuente principal a través de caños o canales meandricos. Estas ciénegas se encuentran localizadas en su mayoría en el sector medio y bajo del río Atrato y se caracterizan por albergar una diversidad de especies de flora y fauna.

Los estudios realizados a la vegetación asociada con ciertos humedales que integran la llanura aluvial del Atrato, registran la presencia aproximada de 382 especies distribuidas en 237 géneros y 67 familias, excluyendo a la vegetación acuática y los pteridofitos (Tabla 1 en anexos). Las familias más representativas corresponden a Arecaceae con 23, Araceae con 21, Fabaceae 19, Rubiaceae 18, Caesalpinaceae 17, Melastomataceae 16, Lecythydaceae 15, Bromeliaceae, Orchidaceae 13 y Bombacaceae con 11 especies (Figura 2).

De las 382 especies presentes en los complejos cenagosos de la llanura aluvial del Atrato, 14 árboles maderables y 1 cícada (Tabla 2), se encuentran en la lista de libros rojos de Colombia (Galeano et al., 2005; Cárdenas y Salinas, 2006; Cogollo et al., 2007), como producto de las fuertes presiones a que han sido sometidos estos ambientes durante largos años, lo cual ha disminuido notablemente las poblaciones de especies de interés biológico y comercial, ocasionando que de algunas de ellas, solo puedan observarse árboles relictuales, pues las poblaciones naturales existentes se encuentran en sitios distantes de difícil acceso.

Desde el punto de vista florístico las áreas boscosas asociadas con los complejos cenagosos de la llanura aluvial del Atrato contienen altos índices de diversidad, que incluyen varias especies como $P$. copaifera, Myroxylon peruiferum, Sterculia apelata, Dipteryx panamensis, Cavanillesia platanifolia, Cordia panamensis, C. pyriformis, Licania duriflora, H. procerum, Huberodendron patinoi, Cariocar amygdaliferum, Zamia chigua restringidas al andén Pacífico, cuyas poblaciones se están fuertemente presionadas por la transformación que han sufrido los diferentes ambientes que conforman la región. Según Aldana (2011) en los bosques de la región zona baja del del Atrato la tasa de deforestación anual corresponde al $0,5 \%$, cifra alta respecto al promedio nacional, situación que pone en riesgo las poblaciones naturales de muchas especies. Estas cifras tienden a aumentar, con la inclusión de prácticas de aprovechamiento y la colonización de nuevas tierras implementación de estas actividades como la minería, realizada en el medio Atrato, aumentan las cifras de deterioro y fragmentación de los bosques de esta unidad paisajística, así como la disminución de la vida silvestre a causa de la desecación de humedales y la contaminación de sus aguas.
Gremios ecológicos. Según las especies asociadas con las ciénagas presentes en la llanura aluvial del río Atrato, se puede determinar que estas conforman 4 gremios ecológicos, cada uno con taxones similares en cuanto a características biológicas y ecológicas, pues comparten aspectos como patrones de regeneración natural, potencial de crecimiento, propiedades de la madera y tipos de uso. Finegan (1993), las define como heliófitas efímeras, heliófitas durables, esciófitas parciales y esciófitas totales. De acuerdo con lo anterior, los gremios con el mayor número de especies asociadas fueron HE con $4 \%$, seguido de HD con $40 \%$, las EP $49 \%$ y las ET $7 \%$.

Las heliófitas efímeras estuvieron conformadas por Ochroma pyramidale, Croton killipianus, Alchornea sp., cuyas capacidades reproductivas y de adaptación a sitios desprovistos de vegetación, les permiten jugar un papel clave en la colonización de zonas abiertas; la tolerancia a la radiación solar y rápido crecimiento, las facultan para colonizar este tipo de ambientes. Las heliófitas duraderas estuvieron representadas por 33 especies, las cuales son intolerantes a la sombra, de rápido crecimiento y ciclo de vida relativamente largo. Su estrategia biológica es similar a la de las HE, en términos menos extremos. Tienen facultadas para poblar espacios de variados tamaños; este grupo reemplazan a las efímeras y se establecen por un período más largo; las especies más dominantes en estos ambientes corresponden a Cecropia spp., Inga spp., Apeiba spp., Vochysia ferruginea, V.jefensis, H. patinoi, C. odorata, Cespedecia macrophilla, C. spatulata, Pachira acuatica. Las especies de este grupo se caracterizan por dispersión anemófila principalmente, con semillas de tamaño variable (pequeñas a grandes), de vida corta, son escasas en el banco de semillas, sus plántulas son capaces de sobrevivir un año (CONIP y PIZANO, 1999). Las esciófitas permanentes estuvieron representadas por 41 especies donde sobresalen Brosimum utile, Campnosperma panamense, Carapa guianensis, C. pyriformis, Cynometra martiana, P. copaifera, Eschweilera integrifolia, Eugenia acapulcensis, Pentaclethra macroloba, Pouteria sp.; este grupo es tolerante a la sombra en las etapas tempranas de su desarrollo, no obstante requieren elevadas tasas de iluminación para pasar de las etapas intermedias a la madurez; soportan la sombra pero no la requieren y aumentan el crecimiento si se abre el dosel del bosque permitiendo mayor ingreso de luz solar. Aunque tienen la capacidad de incrementar su crecimiento al aumentar la radiación, su ciclo de vida es centenario (100 o más años), pues destinan sus recursos principalmente a conformar estructuras más sólidas y duraderas que las de las heliófitas (madera y corteza), soportan condiciones adversas, como la sombra o el suelo inundado $(P$. copaifera). Muchas de las especies que conforman este gremio, tienen semillas apetecidas por la fauna silvestre, sobre todo los roedores, los cuales sirven como mecanismo 
Vegetación vascular y los complejos cenagosos. Z. Valoyes-Cardozo, G. Ramírez-Moreno

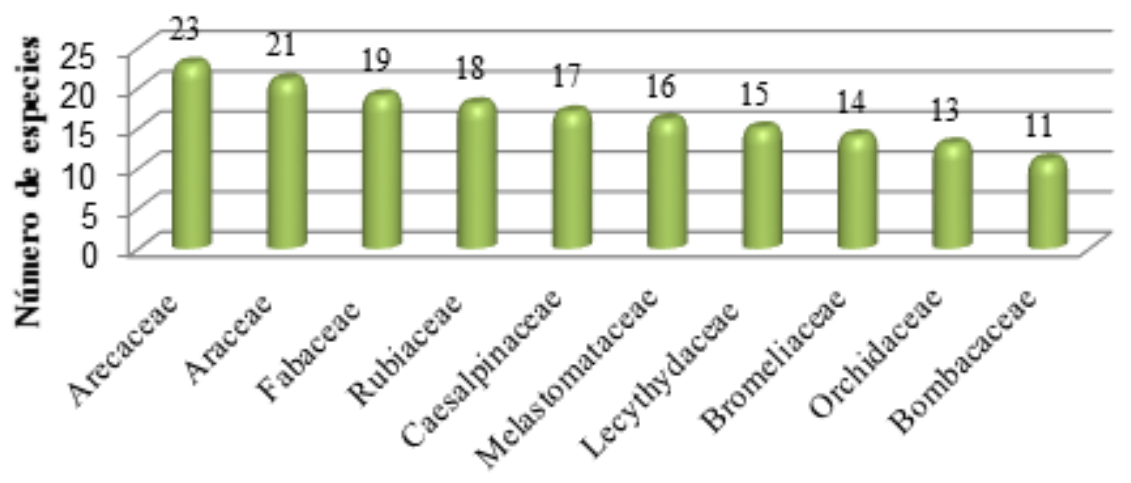

Familias Botánicas

\section{Figura 2. Familias representativas de la vegetación asociada con los complejos cenagosos en la llanura aluvial del río Atrato}

de dispersión, algunas escapan del consumo logrando germinar y así ayudan a la repoblación (CONIP y PIZANO, 1999). Por su parte el gremio de las esciófitas totales estuvo representado por 6 especies, equivalentes al 7\% Brosimum guianensis, Chrysophillum superbum, C. oleafolim, $H$. procerum, Minquartia guianensis, Pouteria caimito; este grupo es relativamente reducido comparado con las EP; requieren sombra y no tienen la capacidad de aumentar significativamente su crecimiento con la apertura del dosel. Muchos de los aspectos de biología y ecología de las EP son válidos también para las esciófitas totales (CONIP y PIZANO, 1999;BELFOR, 2003).

La extracción forestal en épocas anteriores fue una actividad extremadamente selectiva y enfocada principalmente a unas cuantas especies ( $P$. copiafera, $H$. procerum, $C$. piriformis, C. odarata, A. excelsum, Lecythis ampla), al disminuir las poblaciones naturales de estas especies, se incrementó la extracción a un grupo menos conocido, dando paso a especies de poco valor comercial. La inclusión de actividades económicas como la siembra de monocultivos, la ganadería y la minería a los ecosistemas boscosos que circundan los complejos de humedales, están causando dificultades para la regeneración adecuada de varias de estas, restringiéndolas a sitios de difícil acceso.

A pesar que las especies asociadas con estos humedales, desempeñan un papel fundamental en la provisión de hábitat y alimento a la fauna residente y transitoria, la fragmentación y en ocasiones eliminación del bosque ha ocasionado la reducción y cambios en las poblaciones de estas. Linares (1998) y Gonzáles (1991) manifiestan que la extracción selectiva disminuyó notablemente los rodales puros de cativo cuya área inicial cubría unas 363.000 ha hacia la parte baja del Atrato; en la actualidad no se precisa el área total de estas poblaciones, las cuales fueron diezmadas y remplazadas por pastizales para la cría de ganado, así como la siembra de monocultivos de palma aceitera, eliminando las posibilidades de resiliencia de la especie en estos ambientes.

En relación con la problemática expuesta, los datos obtenidos por el IIAP (2002, 2009, 2012 y 2013) en áreas boscosas que circundan los humedales de la zona media del Atrato, muestran que a pesar de los efectos causados por las actividades de uso extractivo practicadas sobre la vegetación asociada con los complejos cenagosos que integran la llanura aluvial del Atrato, aún existen humedales con áreas de bosque conservadas a moderadamente intervenidas. Exhibiendo una estructura con un estrato herbáceo ralo, en donde predominan especies como Monotagma laxon, Maclenia smithiana, Displasia karatifolia, Fimbristilis annua, Mapania sylvatica, acompañadas de una amplia gama de Pteridophytos distribuidos desde el suelo hasta el dosel. El sotobosque alberga especies como T. guianensis, Miconia sp., Chamaedorea sp., Manicaria saccifera, Geonoma triandra, Geonoma sp., Piper arborium, entre otras. El estrato arbóreo lo conforman especies asociadas con diferentes gremios ecológicos $H$. patinoi, C. spathulata, Vochysia jefensis, Calophyllum longifolium, A. aspera, P. macroloba, P. copaifera, C. guinensis, Sloanea zuluaensis, H. procerum, Eschweilera pittieri, asociada con estas se encuentran D. panamensis, Mabea occidentalis, Beilschmiedia rohliana, Protium veneralense, Eugenia lineatifolia, Tapirira myrianthus, $M$. castano, Ocotea cernua, Guateria sp., Aniba sp., Ambalia sp., Pera arborea, Molovetia sp., Sclerolobium sp., Couma macrocarpa, Pouteria sp., Astrocaryum standleyanum, Cynometra martiana. De igual forma estos estudios muestran que en ambientes fuertemente intervenidos la estructura y composición de la vegetación cambia notablemente; el estrato arbóreo es dominada por $C$. spathulata, palmas (Oenocarpus bataua, Oenocarpus minor, Welfia regia, Wetinia quinaria, Mauritiella macroclada).

La composición y arreglo de estas especies cambia según el área de ubicación de los complejos cenagosos dentro de la llanura aluvial y el grado de intervención que estos presenten; 
Bioetnia Volumen 9 № 2 (julio-diciembre), 2012

Tabla 2

Especies Amenazadas presentes en la Ilanura aluvial del Atrato

\begin{tabular}{llll}
\hline Familia & \multicolumn{1}{c}{ Nombre científico } & Nombre Vulgar & Categoría de amenaza \\
\hline \multirow{2}{*}{ Anacardiaceae } & Campnosperma panamense & Sajo & NT \\
& Anacardium excelsum & Caracolí & $\mathrm{NT}$ \\
Bombacaceae & Huberodendron patinoi & Carra & $\mathrm{VU}$ \\
Caesalpinaceae & Prioria copaifera & Cativo & $\mathrm{EN}$ \\
& Orphanodendrum bernalii & Tirateté & $\mathrm{VU}$ \\
Cariocariaceae & Cariocar amygdaliferum & & $\mathrm{VU}$ \\
Crysobalanaceae & Licania duriflora & Carbonero & $\mathrm{EN}$ \\
Fabaceae & Hymenaea oblongifolia & Algarrobo & $\mathrm{NT}$ \\
Humiriaceae & Humiriastrum procerum & Chano & $\mathrm{CR}$ \\
Lauraceae & Aniba perutilis & Chachajo & $\mathrm{CR}$ \\
Lecythidaceae & Cariniana pyriformis & Abarco & $\mathrm{CR}$ \\
Meliaceae & Cedrela odarata & Cedro & $\mathrm{VU}$ \\
& Carapa guianensis & Huino & $\mathrm{EN}$ \\
Myristicaceae & Otoba lehemannii & Cuanguare & $\mathrm{VU}$ \\
Zamiaceae & Zamia chigua & Chigua & $\mathrm{VU}$ \\
\hline
\end{tabular}

hacia la parte baja del Atrato, donde la vegetación existente que integra el bosque asociado con los humedales es más homogénea por la condición de bosque inundable conformado por asociaciones singulares integradas por especies particulares casi homogéneas como panganales (Raphia taedigera), cativales ( $P$. copaifera). Estos grupos, han estado sometidos a diferentes presiones, situación que dependiendo del tipo de disturbio, impide la resiliencia del ambiente, cambiando en algunos casos la composición original, permitiendo la inclusión de otras especies ocasionando que asociaciones casi homogéneas como los cativales, en la actualidad estén formadas por otras especies como A. exelsum, C. guianensis, Tabebuia rosea, Cecropia spp.; en las ciénagas que se encuentran bajo la influencia marina la composición y estructura cambia notablemente, dando paso a especies con otros requerimientos ecológicos (Rhizophora mangle), la cual a su vez se combinan con elementos como $R$. taedigera, Pterocarpus officinalis, P. copaifera, cuyas poblaciones originales han sido reemplazadas por el establecimiento de pastos para la ganadería (CODECHOCO y CORPOARIEN, 2011).

Los temas tratados permiten que se establezcan diferencias entre una zona y otra; Ramírez y Valoyes (2009) reportan que la ciénaga Grande de Beté ubicada en la zona del medio Atrato, comparada con otros complejos cenagosos de la misma localidad, muestren que este es un ecosistema en buen estado de conservación, con un esqueleto vegetal complejo y una vegetación diversa, lo cual hace que este ambiente se convierta en el espacio propicio de organismos que habitan allí de forma permanente (endemismos y restricciones), además de ser el hábitat predilecto de otros que viajan grandes distancias solo para completar un ciclo de su vida (migratorios) repitiendo esta proeza temporada tras temporada.

Por otro lado, el IIAP (2012) manifiesta que la conservación de los bosques asociados con los diferentes complejos cenagosos, varía de una localidad a otra, dependiendo de la vocación de las comunidades asentadas en los alrededores de estos ecosistemas. El esqueleto vegetal de los bosque que rodean el complejo cenagoso La Larga, varía notablemente en su composición y estructura florística, pues se evidencia la degradación que han sufrido estos bosques, a causa de la constante e intensa explotación forestal, situación que se evidencia con la estratificación del bosque conformado principalmente por un estrato herbáceo en donde predominan especies generalistas, con un sotobosque casi ausente, pues predominan especies de rápido crecimiento (C. spatulatha, Miconia spp.), este da paso a un estrato arbóreo conformado principalmente por palmas (Wettinia quinaria, O. bataua, $O$. minor, Welfia regia, M. macroclada) que, aunque generalmente son plantas de dosel medio soportan espacios abiertos con alta intensidad lumínica. Yepes et al. (2010) manifiestan que $O$. bataua es una especie típica indicadora de bosques primarios; la alta ocurrencia de esta especie en ambientes altamente intervenidos de la llanura aluvial del Atrato, puede estar relacionada con la preferencia de los pobladores por las 
especies maderables, por lo cual las palmas porte arbóreo son dejadas, convirtiéndose en la vegetación que compite por la dominancia del dosel, ante la ausencia de especies de importancia biológica y comercial.

Semejante es el grado de afectación de los bosques que circundan la ciénaga la Honda-Tanguí, en donde el IIAP (2013) reporta que la vegetación arbórea aledaña a este ecosistema, fue extraída con fines comerciales, situación que ha afectado estos ambientes disminuyendo la riqueza de especies de importancia ecológica y ha dado paso a especies generalistas (Condaminea corymbosa, Isertia sp., C. spatulatha, Sauvagesia erecta Pentagonia macrophylla, Psychotria cinta, Psychotria poepigiana, Psychotria racemosa, Psychotria sp., T. guinensis, Miconia pileata, Faramea multiflora, Faramea ocidentalis, Faramea sp., Aciotis sp., Cecropia spp., Paulinia spp.). Situación que se percibe aún más en las ciénagas de la parte baja del río Atrato, donde IIAP (2002) en una evaluación de las ciénagas Tumarado y Perancho, la Honda y la Rica, reporta la baja representación de especies, ocasionada por las condiciones ambientales y la fuerte presión que se ejerció durante décadas sobre los bosques adyacentes a estos ecosistemas; las especies predominantes corresponden a especies de poca importancia comercial. Aunque aún se conservan remanentes de bosque de P. copaifera asociados con Pachira acuatica, M. macroclada, Astrocariun standleyanum, Tabebuia sp., Cecropia peltata, Calophylum mariae, Ficus sp., Cynometra longifolium, $C$. guinensis, Inga sp. y P. officinalis, igualmente se conservan Montrichardia arborescens, $R$. taedigera asociadas con especies herbáceas de poco valor comercial. En relación con la temática expuesta, autores como Castillo-Cortés y Gonzáles (2002) manifiestan que a pesar del deterioro de la cobertura boscosa generalizado en la zona baja del Atrato, en algunas áreas asociadas con los humedales, se logra diferenciar una mezcla de elementos de tierras bajas de la región Caribe y Pacífico, situación que hace que estas ciénegas representen áreas con atributos importante para la conservación de la fauna silvestre.

En relación con la temática expuesta se puede asegurar que en la zona baja de la llanura aluvial del Atrato, diferentes actividades como la siembra de pastos para la ganadería, el monocultivo de la palma aceitera, la agricultura extensiva, los cultivos ilícitos y la intensiva extracción forestal transformaron la vegetación arbórea original, aunque aún se conservan remanentes de bosques de cativo, asociado con ciertas especies de importancia comercial. Por lo anterior (CODECHOCO y CORPOURABA, 2006) deducen que es casi inexistente la vegetación de los humedales del bajo Atrato correspondientes a Tumarado, Marriaga y Unguía, atribuyendo los anteriores argumentos a los incendios forestales producidos en la década de 1990. Pese a lo anterior, CODECHOCÓy CORPARIEN(2011)manifiestan que elcom- plejo Marriaga-Limón por su cercanía al mar presentan una vegetación con una composición y estructura que varía con respecto a los humedales referenciados en la llanura aluvial del Atrato, en este ecosistema se combinan diferentes asociaciones como manglares, cativales, panganales y arracachales, hacia las zonas de colinas bajas predomina una vegetación heterogénea con especies como C. odorata, A. excelsum, Calophylum mariae, Ficus sp., Cynometra longifolium, $C$. guinensis, Inga sp., y $P$. officinalis, especies que en la actualidad se encuentran dispersas, pues la vegetación original fue reemplazada por pastizales para el sostenimiento de la ganadería, de la misma forma se plantaron monocultivos y agricultura extensiva, fragmentando el bosque y disminuyendo la cobertura boscosa, que desaparece a un ritmo alarmante.

Los cambios en el clima ha contribuido a los cambios ocurridos a la vegetación que circunda los complejos cenagosos de la llanura aluvial del Atrato, pues la alteración de la dinámica del río cambia algunas características biológicas y fisiológicas de la vegetación que integran estos ambientes, permitiéndoles algunas modificaciones fisiológicas que permitan a las plantas adaptarse a las condiciones extremas presentes en este medio. CONIF y PIZANO (1999) manifiestan que las adaptaciones de estos grupos, obedecen a un largo proceso de evolución natural, cuya culminación es una forma de organización, funcionamiento e interrelación de los componentes, por lo cual, los cambios introducidos por factores exógenos producen cambios que alteran radicalmente ese modelo natural. La anterior afirmación es consecuente con los cambios que han tenido los bosques de la llanura aluvial del Atrato, en donde se evidencia los cambios ocurridos en poblaciones como los cativales, los cuales han modificado su composición, pasaron de ser asociaciones casi homogéneas que en la antigüedad se caracterizaban por formar rodales puros, a ser bosques heterogéneos. Muchos de estos fueron reemplazados por áreas ganaderas, situación que ha causado efectos en la dinámica de los humedales asociados con ellos.

En este mismo contexto, los cambios ocasionados a la vegetación de esta unidad paisajística como el reemplazado por monocultivos y plantaciones forestales, han consentido que hoy se deduzcan los cambios que las actividades mencionadas antes han causado en esta unidad paisajística, lo cual es fácilmente apreciable por la disminución de la diversidad local y alteración de la distribución natural de ciertas especies. Hofstede et al. (1998) manifiestan que las plantaciones de especies exóticas puede ser de mucho beneficio para la industria maderera y las comunidades rurales, pero al mismo tiempo que causan impactos sobre el recurso existente, aunque no hay absoluta claridad sobre los efectos que causan estas actividades sobre ecosistemas naturales, hay más indicaciones de deterioro que de recuperación de los ambientes donde se efectúa el cambio, los cuales se reflejan en la hidrología, la fertilidad de los suelos y la diversidad. 


\section{Bioetnia Volumen 9 № 2 (julio-diciembre), 2012}

Estas afirmaciones son corroboradas por Carrasco y Flores (2002) quienes aseguran que el monocultivo de la palma aceitera se ha naturalizado gracias a sus mecanismos de dispersión (zoocora por aves y mamíferos e hidrocora), extendiéndose en un $95 \%$ en los litorales lagunares, planicies de inundación y humedales marinos en el Caribe.

Lo anterior representa un peligro inminente para la diversidad que sustentan los bosques de esta región, en los cuales las comunidades asentadas en los alrededores de los ecosistemas cenagosos referenciados, basan su economía debido a los bienes que estos proporcionan (madera, peces, fauna de cacería y productos no maderables del bosque). A pesar del deterioro que estos han venido sufriendo, pues en las últimas décadas se han intensificado diferentes actividades que requieren la eliminación parcial y en algunos casos total de la vegetación natural de estos ambientes, fragmentando las selvas, disminuyendo así el desplazamiento de la fauna circundante por la ruptura de estos corredores, por consiguiente a medida que se pierde la cobertura boscosa, disminuyen los recursos que estos proporcionan y se intensifican los esfuerzos por mantener una economía de subsistencia, generando así conflictos sociales y graves problemas ambientales.

\section{Conclusiones}

Los datos obtenidos ponen de manifiesto que la vegetación asociada con los complejos cenagosos en la zona media y baja de la llanura aluvial del río Atrato, presenta diferentes grados de intervención, lo cual ha modificado la composición original, la estructura y la dinámica de estos ambientes. Los registros florísticos referenciados permiten deducir que existen diferencias en cuanto a la composición y arreglos florísticos en estas dos áreas de la unidad paisajística; se observa también que la intensidad de las actividades antrópicas que se han desarrollado en cada una de ellas varia en intensidad; la vegetación asociada con los complejos cena- gosos de la zona media del Atrato esta mejor conservada que la parte baja del mismo río; las evidencias muestran que los disturbios por las diferentes actividades han causado mayores impactos hacia la parte baja de la llanura aluvial, aunque esta zona se atribuye una alta diversidad, la vegetación tiende a ser más homogénea producto de las extensas áreas formadas por las diferentes asociaciones allí conformadas.

A pesar de los vacíos de información relacionados con la vegetación y la flora de los bosques aledaños a la llanura aluvial del Atrato, existen evidencias que ponen de manifiesto la perdida de la diversidad contenida en estos. La información disponible permite inferir que las actividades que se han desarrollado han causado daños incalculables, las cuales se han desarrollado con mayor intensidad hacia la zona baja del río Atrato; sin embargo el deterioro que se ha estado causando en el medio Atrato, específicamente por actividades como la minería en los ríos Bebara y Bebarama, y el aumento de cultivos de palma aceitera, yuca, plátano y ganadería en los ríos Curvaradó y Jiguamiando (Carmen del Darién) crea una alerta que debe ser atendida de forma inmediata por la importancia ecológica, económica y cultural de estos ecosistemas.

Las ideas expuestas ponen de manifiesto la necesidad de aplicar las diferentes estrategias planteadas por parte de las corporaciones que tiene injerencia en el área de influencia de estos humedales; la problemática ambiental cada vez se hace más notoria, con la disminución de la cobertura boscosa y el agotamiento de especies de interés especial. La disminución de la cobertura boscosa disminuye la oferta alimenticia, por lo cual disminuye la fauna residente que migra a otros espacios con los requerimientos necesarios para su sostenimiento y la estabilidad de sus poblaciones. Lo anterior es un llamado de atención y una alerta a las autoridades ambientales a realizar estudios de caracterización y análisis de los recursos existentes en estos ecosistemas, los cuales son esenciales para la toma de decisiones y puesta en marcha de acciones que ayuden a la restauración de estos ambientes. 
Vegetación vascular y los complejos cenagosos. Z. Valoyes-Cardozo, G. Ramírez-Moreno

Tabla 1

Especies presentes en los ciénegas de la llanura aluvial del Atrato

\begin{tabular}{|c|c|c|c|c|c|c|c|}
\hline \multirow[t]{3}{*}{ Familia } & \multirow[t]{3}{*}{ Especie } & \multicolumn{6}{|c|}{ Complejos cenegosos } \\
\hline & & \multicolumn{2}{|c|}{ La Grande La Larga } & \multirow{2}{*}{$\begin{array}{l}\text { La Honda } \\
\text { Tanguí }\end{array}$} & \multirow{2}{*}{$\begin{array}{c}\text { Marriaga } \\
\text { Limón }\end{array}$} & \multirow{2}{*}{$\begin{array}{l}\text { Tumarado } \\
\text { Perancho }\end{array}$} & \multirow{2}{*}{$\begin{array}{l}\text { Gremio } \\
\text { Ecológico }\end{array}$} \\
\hline & & Beté & Tagachí & & & & \\
\hline \multirow[t]{6}{*}{ Anacardiaceae } & Anacardium excelsum & $\mathrm{x}$ & & & $x$ & & HD \\
\hline & Astronium graveolens & & & & & $x$ & HD \\
\hline & Tapirira guianensis & $x$ & & & & & \\
\hline & Canosperma sp & & & $\mathrm{X}$ & & & \\
\hline & Campnosperma panamense & $\mathrm{x}$ & $x$ & & $\mathrm{x}$ & & EP \\
\hline & Spondias mombin & & $\mathrm{x}$ & & & & $\mathrm{HD}$ \\
\hline \multirow[t]{10}{*}{ Anonaceae } & Anaxagorea crassipetala & & & $\mathrm{x}$ & & & \\
\hline & Annona glabra & & & $\mathrm{x}$ & & & EP \\
\hline & Guatteria amplifolia & & & $\mathrm{x}$ & & & \\
\hline & Guateria cargadero & $\mathrm{x}$ & $\mathrm{X}$ & $\mathrm{x}$ & & & \\
\hline & Guateria chocoensis & $\mathrm{X}$ & & & & & \\
\hline & Fusaea longifolia & $\mathrm{x}$ & & & & & \\
\hline & Rollinia mucosa & $\mathrm{X}$ & & & & & \\
\hline & Rollinia pittieri & $\mathrm{X}$ & & & & & \\
\hline & Unonopsis velutina & & & & $\mathrm{x}$ & & HD \\
\hline & Xylopia sp & $\mathrm{x}$ & & & & $x$ & \\
\hline \multirow[t]{9}{*}{ Apocynaceae } & Allamanda cathartica & $\mathrm{x}$ & & & & & \\
\hline & Allomarkgrafia ovalis & & & $\mathrm{x}$ & & & \\
\hline & Ambelia sp & $\mathrm{x}$ & & & & & \\
\hline & Aspidosperma sp & & & $\mathrm{x}$ & & & \\
\hline & Couma macrocarpa & $\mathrm{x}$ & $X$ & $\mathrm{x}$ & & & \\
\hline & Himatanthus sp & & & $\mathrm{x}$ & & & \\
\hline & Lamacella floribunda & $\mathrm{x}$ & & & & & \\
\hline & Lamacella panamensis & $\mathrm{X}$ & & & & & \\
\hline & Malovetia $s p$ & $\mathrm{X}$ & $\mathrm{X}$ & & & & \\
\hline Asteraceae & Ambrosia peruviana & & & & $\mathrm{x}$ & & \\
\hline \multirow[t]{3}{*}{ Araliaceae } & Dendropanax arboreum & $\mathrm{x}$ & & & & & \\
\hline & Scheflera $s p$ & $\mathrm{x}$ & $X$ & $\mathrm{x}$ & & & \\
\hline & Schefflera minutiflora & & & $\mathrm{X}$ & & & \\
\hline \multirow[t]{9}{*}{ Araceae } & Anthurium fragantisimun & $\mathrm{x}$ & & $\mathrm{x}$ & & & \\
\hline & Anthurium formosum & $\mathrm{X}$ & & $\mathrm{X}$ & & & \\
\hline & Anthurium obtusum & $\mathrm{x}$ & $\mathrm{X}$ & & & & \\
\hline & Anthurium bakeri $\mathrm{H}$ & $\mathrm{x}$ & & & & & \\
\hline & Anthurium hacumense & & & $\mathrm{x}$ & & & \\
\hline & Anthurium cf longistipitatum & & & $\mathrm{x}$ & & & \\
\hline & Anthurium silvanii & $\mathrm{x}$ & $X$ & $\mathrm{x}$ & & & \\
\hline & Anthurium scandens & $\mathrm{x}$ & & & & & \\
\hline & Anthurium vallense & & & $\mathrm{X}$ & & & \\
\hline
\end{tabular}


Bioetnia Volumen 9 № 2 (julio-diciembre), 2012

Tabla 1

Especies presentes en los ciénegas de la llanura aluvial del Atrato

\begin{tabular}{|c|c|c|c|c|c|c|c|}
\hline \multirow[t]{3}{*}{ Familia } & \multirow[t]{3}{*}{ Especie } & \multicolumn{5}{|c|}{ Complejos cenegosos } & \multirow{3}{*}{$\begin{array}{c}\text { Gremio } \\
\text { Ecológico }\end{array}$} \\
\hline & & \multicolumn{2}{|c|}{ La Grande La Larga } & \multirow{2}{*}{$\begin{array}{l}\text { La Honda } \\
\text { Tanguí }\end{array}$} & \multirow{2}{*}{$\begin{array}{c}\text { Marriaga } \\
\text { Limón }\end{array}$} & \multirow{2}{*}{$\begin{array}{l}\text { Tumarado } \\
\text { Perancho }\end{array}$} & \\
\hline & & Beté & Tagachí & & & & \\
\hline & Dracontium grayumianum & & & $x$ & & & \\
\hline & Monstera $s p$ & $x$ & $x$ & $x$ & & & \\
\hline & Montrichardia arborescens & $x$ & $x$ & & $x$ & $x$ & \\
\hline & Philodendron fragantissimum & $x$ & $x$ & $x$ & & & \\
\hline & Philodendron heleniae & $x$ & $x$ & & & & \\
\hline & Philodendron squamiferum & & $x$ & & & & \\
\hline & Philodendron ssp & $x$ & & $x$ & & & \\
\hline & Spathiphylum friedrichsthalii & $x$ & $x$ & $x$ & & & \\
\hline & Spathiphyllum clevelandii & & $x$ & & & & \\
\hline & Spathiphylum sp & & $x$ & & & & \\
\hline & Stenospermation angustifolium & & & $x$ & & & \\
\hline & Stenospermation $s p$ & & & & & & \\
\hline \multirow[t]{23}{*}{ Arecaceae } & Astrocaryum standleyanum & & & & & & \\
\hline & Asterogyne martiana & & & & & & \\
\hline & Attalea alleni & $x$ & & $x$ & & & \\
\hline & Bactris brongniartii & $x$ & $x$ & $x$ & & & \\
\hline & Bactris sp & & $x$ & $x$ & & & \\
\hline & Camaedorea sp & $x$ & & & & & \\
\hline & Desmoncus cirrhiferus & $x$ & $x$ & & & & \\
\hline & Elaeis panamensis & & & & $x$ & $x$ & \\
\hline & Euterpe precatoria & $x$ & $x$ & $x$ & $x$ & $x$ & \\
\hline & Iriartea deltoidea & $x$ & & $x$ & & & \\
\hline & Geonoma cuneata & & & $x$ & & & \\
\hline & Geonoma deversa & & & $x$ & & & \\
\hline & Geonoma triandra & $x$ & & & & & \\
\hline & Geonoma sp & $x$ & & & & & \\
\hline & Manicaria saccifera & $x$ & $x$ & $x$ & $x$ & $x$ & \\
\hline & Mauritiella macroclada & $x$ & $x$ & $x$ & $x$ & $x$ & \\
\hline & Oenocarpus bataua & $x$ & & $x$ & & $x$ & EP \\
\hline & Oenocarpus minor & $x$ & $x$ & $x$ & & & \\
\hline & Raphia taedigera & & $x$ & $x$ & $x$ & $x$ & \\
\hline & Synechanthus warscewiczianus & & & $x$ & & & \\
\hline & Socratea exorrhiza & $x$ & & $x$ & & & EP \\
\hline & Wetinia quinaria & $x$ & & $x$ & & & EP \\
\hline & Welfia regia & $x$ & & $x$ & & & \\
\hline Asclepidaceae & Sarcostemmma sp & $x$ & & & & & \\
\hline \multirow[t]{2}{*}{ Bignoniaceae } & Anemopaegma chamberlaynii & $x$ & $x$ & & & & \\
\hline & Ciclostoma binatum & $\mathrm{x}$ & $x$ & & & & \\
\hline
\end{tabular}


Vegetación vascular y los complejos cenagosos. Z. Valoyes-Cardozo, G. Ramírez-Moreno

Tabla 1

Especies presentes en los ciénegas de la llanura aluvial del Atrato

\begin{tabular}{|c|c|c|c|c|c|c|c|}
\hline \multirow[t]{3}{*}{ Familia } & \multirow[t]{3}{*}{ Especie } & \multicolumn{6}{|c|}{ Complejos cenegosos } \\
\hline & & \multicolumn{2}{|c|}{ La Grande La Larga } & \multirow{2}{*}{$\begin{array}{c}\text { La Honda } \\
\text { Tanguí }\end{array}$} & \multirow{2}{*}{$\begin{array}{c}\text { Marriaga } \\
\text { Limón }\end{array}$} & \multirow{2}{*}{$\begin{array}{r}\text { Tumarado } \\
\text { Perancho }\end{array}$} & \multirow{2}{*}{$\begin{array}{l}\text { Gremio } \\
\text { Ecológico }\end{array}$} \\
\hline & & Beté & Tagachí & & & & \\
\hline & Cryptostegia madagascariencis & & & & $x$ & & \\
\hline & Lundia sp & & & $x$ & & & \\
\hline & Martinella obovata & & & $x$ & & & \\
\hline & Tabebuia rosea & & $x$ & & $x$ & & $\mathrm{HD}$ \\
\hline \multirow[t]{11}{*}{ Bombacaceae } & Cavanillesia platanifolia & & & & & $x$ & \\
\hline & Ceiba $s p$ & & & $x$ & & & \\
\hline & Huberodendron patinoi & $x$ & & $x$ & $x$ & & $\mathrm{HD}$ \\
\hline & Matisia bullata & & & $x$ & & & $\mathrm{EP}$ \\
\hline & Matisia castano & $x$ & & $x$ & & & EP \\
\hline & Matisia pectandra & & $x$ & & & & EP \\
\hline & Matisia sp & & $x$ & $x$ & & & EP \\
\hline & Ochroma pyramidale & $x$ & & $x$ & & $x$ & $\mathrm{HE}$ \\
\hline & Pachira acuatica & $x$ & $x$ & & $x$ & $x$ & $\mathrm{HD}$ \\
\hline & Pachira quintana & & $x$ & $x$ & & & \\
\hline & Pseudobombax septenatum & & & & & $x$ & $\mathrm{HD}$ \\
\hline \multirow[t]{14}{*}{ Bromeliaceae } & Aechmea longicuspis & $x$ & $x$ & $x$ & & & \\
\hline & Aechmea magdalenae & $x$ & & $x$ & & & \\
\hline & Aechmea tillandsioides & & $x$ & & & & \\
\hline & Guzmania calamifolia & & & $x$ & & & \\
\hline & Guzmania glomerata & $x$ & & $x$ & & & \\
\hline & Guzmania lingualata & $x$ & $x$ & $x$ & & & \\
\hline & Guzmania musaica & $x$ & $x$ & $x$ & & & \\
\hline & Guzmania scherzeriana & & & $x$ & & & \\
\hline & Tillandsia anceps & $x$ & & & & & \\
\hline & Tillandsia monadelpha & $x$ & & & & & \\
\hline & Tillandsia bulbosa & $x$ & & $x$ & & & \\
\hline & Racinaea cf tenuispica & $x$ & & & & & \\
\hline & Werauhia sanguinolenta & $x$ & $x$ & $x$ & & & \\
\hline & Werauhia grandiflora & $x$ & $x$ & $x$ & & & \\
\hline \multirow[t]{5}{*}{ Burceraceae } & Protium amplum & & & $x$ & & & \\
\hline & Protium aracouchini & $x$ & & & & & \\
\hline & Protium sagotianum & $x$ & & & & & \\
\hline & Protium inconforme & $x$ & & & & & \\
\hline & Protium $s p$ & & & $x$ & & & EP \\
\hline Caryocaraceae & Caryocar amygdaliferum & $x$ & & & & & \\
\hline \multirow[t]{3}{*}{ Caesalpinaceae } & Bauhinia gabra & & & $x$ & & & \\
\hline & Bauhinia minor & $x$ & & & & & \\
\hline & Cassia grandis & $x$ & & & & & \\
\hline
\end{tabular}


Bioetnia Volumen 9 № 2 (julio-diciembre), 2012

Tabla 1

Especies presentes en los ciénegas de la llanura aluvial del Atrato

\begin{tabular}{|c|c|c|c|c|c|c|c|}
\hline \multirow[t]{3}{*}{ Familia } & \multirow[t]{3}{*}{ Especie } & \multicolumn{6}{|c|}{ Complejos cenegosos } \\
\hline & & \multicolumn{2}{|c|}{ La Grande La Larga } & \multirow{2}{*}{$\begin{array}{c}\text { La Honda } \\
\text { Tanguí }\end{array}$} & \multirow{2}{*}{$\begin{array}{l}\text { Marriaga } \\
\text { Limón }\end{array}$} & \multirow{2}{*}{$\begin{array}{l}\text { Tumarado } \\
\text { Perancho }\end{array}$} & \multirow{2}{*}{$\begin{array}{l}\text { Gremio } \\
\text { Ecológico }\end{array}$} \\
\hline & & Beté & Tagachí & & & & \\
\hline & Cassia reticulata & $x$ & & & & & \\
\hline & Copaifera canime & $x$ & & & & & \\
\hline & Copaifera chiriquen & $x$ & & & & & \\
\hline & Cynometra martiana & & & & & $x$ & EP \\
\hline & Dialimun guianensis & $x$ & $x$ & & & & \\
\hline & Hymeneaea courbaril & $x$ & & & & & EP \\
\hline & Hymeneaea oblogifolia & $x$ & $x$ & $x$ & & & \\
\hline & Hymeneaea palustris & $x$ & & & & & \\
\hline & Orphanodendrum bernalii & $x$ & & & $x$ & & \\
\hline & Prioria copaifera & $x$ & $x$ & & $x$ & $x$ & EP \\
\hline & Senna sp & & & $x$ & & & \\
\hline & Swartzia panamensis & $x$ & & & & & \\
\hline & Swartzia sp & & & $x$ & & & \\
\hline & Sclerolobium $s p$ & $x$ & & & & & \\
\hline Cactaceae & Epiphyllum phyllanthus & $x$ & $x$ & & & & \\
\hline \multirow[t]{6}{*}{ Cecropiaceae } & Cecropia insignis & $x$ & & & & & $\mathrm{HD}$ \\
\hline & Cecropia longipes & $\mathrm{x}$ & & & & $x$ & $\mathrm{HD}$ \\
\hline & Cecropia obtusifolia & $x$ & & & & & $\mathrm{HD}$ \\
\hline & Cecropia membranacea & $x$ & & & & & $\mathrm{HD}$ \\
\hline & Cecropia peltata & & $x$ & & & & $\mathrm{HD}$ \\
\hline & Pouruma bicolor & $x$ & & & & & \\
\hline \multirow[t]{4}{*}{ Costaceae } & Costus scaber & $x$ & & & & & \\
\hline & Costus spiralis & $x$ & & & & & \\
\hline & Costus sp & & $x$ & $x$ & & & \\
\hline & Dimerocostus strobilaceus & $x$ & & & & & \\
\hline Combretaceae & Laguncularia racemosa & & & & $x$ & & \\
\hline \multirow[t]{10}{*}{ Clusiaceae } & Calophylum mariae & $x$ & $\mathrm{x}$ & & & & $\mathrm{HD}$ \\
\hline & Calophyllum sp & $x$ & & & & $x$ & $\mathrm{HD}$ \\
\hline & Clusia sp & $x$ & $\mathrm{x}$ & $x$ & & & \\
\hline & Garcinia madrunno & & $\mathrm{x}$ & $x$ & & & EP \\
\hline & Syphonia globulifera & & $\mathrm{x}$ & $x$ & $x$ & $x$ & \\
\hline & Symphonia sp & & & $x$ & & & \\
\hline & Tovomita guianensis & & & $x$ & & & EP \\
\hline & Tovomita stylosa & $x$ & & & & & EP \\
\hline & Rheedia $s p$ & $\mathrm{x}$ & & & & & \\
\hline & Vismia latisepala & $x$ & $x$ & & & & $\mathrm{HD}$ \\
\hline \multirow[t]{2}{*}{ Cyperaceae } & Diplasia karatifolia & $x$ & $x$ & $x$ & & & \\
\hline & Fimbristilis annua & $\mathrm{x}$ & & & & & \\
\hline
\end{tabular}


Vegetación vascular y los complejos cenagosos. Z. Valoyes-Cardozo, G. Ramírez-Moreno

Tabla 1

Especies presentes en los ciénegas de la llanura aluvial del Atrato

\begin{tabular}{|c|c|c|c|c|c|c|c|}
\hline \multirow[t]{3}{*}{ Familia } & \multirow[t]{3}{*}{ Especie } & \multicolumn{6}{|c|}{ Complejos cenegosos } \\
\hline & & \multicolumn{2}{|c|}{ La Grande La Larga } & \multirow{2}{*}{$\begin{array}{c}\text { La Honda } \\
\text { Tanguí }\end{array}$} & \multirow{2}{*}{$\begin{array}{l}\text { Marriaga } \\
\text { Limón }\end{array}$} & \multirow{2}{*}{$\begin{array}{l}\text { Tumarado } \\
\text { Perancho }\end{array}$} & \multirow{2}{*}{$\begin{array}{l}\text { Gremio } \\
\text { Ecológico }\end{array}$} \\
\hline & & Beté & Tagachí & & & & \\
\hline & Mapania sy/vatica & $x$ & & $x$ & & & \\
\hline & Oxycaryum cubense & & & & $x$ & & \\
\hline & Scirpus sp & & $x$ & & & & \\
\hline & Scleria melaleuca & & & & $x$ & & \\
\hline & Scleria secans & & & & & $x$ & \\
\hline & Scleria pterota & & & & & $x$ & \\
\hline & Cyperus luzulae & $x$ & & & & & \\
\hline & Cyperus spp. & & & & & & \\
\hline \multirow[t]{7}{*}{ Cyclanthaceae } & Asplundia rigida & $x$ & $x$ & $x$ & & & \\
\hline & Asplundia sp & & & $x$ & & & \\
\hline & Cyclanthus bipartitus & $x$ & $x$ & $x$ & & & \\
\hline & Evodianthus funifer & $x$ & $x$ & $x$ & & & \\
\hline & Sphaeradenia crocea & & & $x$ & & & \\
\hline & Sphaeradenia killipi & & & & & & \\
\hline & Sphaeradenia stenosperma & & & $x$ & & & \\
\hline \multirow[t]{2}{*}{ Chrysobalanaceae } & Licania sp & & $x$ & & & & \\
\hline & Licania durifolia & & & $x$ & & & \\
\hline \multirow[t]{2}{*}{ Dileniaceae } & Tetracera willdenowiana & & & $x$ & & & \\
\hline & Davilla kunthii & & & $x$ & & & \\
\hline Eleocarpaceae & Sloanea zuliaensis & $x$ & & & & & EP \\
\hline \multirow[t]{3}{*}{ Ericaceae } & Cavendischia sp & $x$ & & & & & \\
\hline & Cavendischia completens & $x$ & & & & & \\
\hline & Maclania smithiana & $x$ & & & & & \\
\hline \multirow[t]{10}{*}{ Euphorbiaceae } & Alchornea $s p$ & & & $x$ & & & $\mathrm{HE}$ \\
\hline & Acalypha $s p$ & & & $x$ & & & \\
\hline & Cariodendrum orinocense & $x$ & & & & & \\
\hline & Croton killipianus & & & $x$ & & & $\mathrm{HE}$ \\
\hline & Hura crepitans & & & & & $x$ & \\
\hline & Mabea chocoensis & & & $x$ & & & \\
\hline & Mabea occidentalis & $x$ & & $x$ & & & \\
\hline & Pera arbórea & $x$ & & & & & \\
\hline & Pera sp & & & $x$ & & & \\
\hline & Pausandra trianeii & $x$ & & & & & \\
\hline \multirow[t]{5}{*}{ Fabaceae } & Abrus fruticulosus & & & $x$ & & & \\
\hline & Acosmium sp & & & & & & \\
\hline & Andira inermis & $x$ & & & & & \\
\hline & Andira $s p$ & & & $x$ & & & \\
\hline & Brownea ariza & & & $\mathrm{x}$ & & & \\
\hline
\end{tabular}


Bioetnia Volumen 9 № 2 (julio-diciembre), 2012

Tabla 1

Especies presentes en los ciénegas de la llanura aluvial del Atrato

\begin{tabular}{|c|c|c|c|c|c|c|c|}
\hline \multirow[t]{3}{*}{ Familia } & \multirow[t]{3}{*}{ Especie } & \multicolumn{6}{|c|}{ Complejos cenegosos } \\
\hline & & \multicolumn{2}{|c|}{ La Grande La Larga } & \multirow{2}{*}{$\begin{array}{l}\text { La Honda } \\
\text { Tanguí }\end{array}$} & \multirow{2}{*}{$\begin{array}{l}\text { Marriaga } \\
\text { Limón }\end{array}$} & \multirow{2}{*}{$\begin{array}{l}\text { Tumarado } \\
\text { Perancho }\end{array}$} & \multirow{2}{*}{$\begin{array}{l}\text { Gremio } \\
\text { Ecológico }\end{array}$} \\
\hline & & Beté & Tagachí & & & & \\
\hline & \multicolumn{2}{|c|}{ Erythrina costaricensis vr panamensis } & $x$ & & & & \\
\hline & \multicolumn{2}{|c|}{ Erythrina fusca } & & & & $x$ & \\
\hline & Desmodium orbiculare & $x$ & & & & & \\
\hline & Dipterix panamensis & $x$ & $x$ & $x$ & $x$ & & \\
\hline & Dussia lehmanii & $x$ & & & & & \\
\hline & Dioclea guianensis & & & $\mathrm{x}$ & & & \\
\hline & Neptunia oleracea & & & & & & \\
\hline & Macrolobium angustifolium & & & $x$ & & & \\
\hline & Muellera sp & $x$ & & & & & \\
\hline & Myroxylon peruiferum & & & & & $x$ & $\mathrm{HD}$ \\
\hline & Oiciocarpum panamensis & $x$ & & & & & \\
\hline & Ormosia $s p$ & & $x$ & & & & \\
\hline & Pterocarpus officinalis & $x$ & & & $x$ & $x$ & $\mathrm{HD}$ \\
\hline & Banara sp & & & $x$ & & $x$ & \\
\hline Fagaceae & Quercus bumboldtii & & & & $x$ & & \\
\hline \multirow[t]{4}{*}{ Flacurtiaceae } & Caseariasylvestris & $x$ & & & & & \\
\hline & Carpotroche aculeata & & & $x$ & & & \\
\hline & Carpotroche pacifica & $x$ & & & & & \\
\hline & Lindackeria sp & & $x$ & & & & \\
\hline \multirow[t]{6}{*}{ Gesneriaceae } & Columnea sp & & $\mathrm{x}$ & $\mathrm{x}$ & & & \\
\hline & Columnea consanguinea & & & $x$ & & & \\
\hline & Columnea crassifolia & $x$ & $x$ & & & & \\
\hline & Columnea picta & & & $x$ & & & \\
\hline & Codonanthe crassifolia & $x$ & & & & & \\
\hline & Drimonia serrulata & $x$ & & & & & \\
\hline \multirow[t]{4}{*}{ Heliconiaceae } & Heliconia $s p$ & & $x$ & & & & \\
\hline & Heliconia lathispata & $x$ & $x$ & & & & \\
\hline & Heliconia regalis & $x$ & & & & & \\
\hline & Heliconia marginata & & & & $x$ & & \\
\hline \multirow[t]{2}{*}{ Humiriaceae } & Humiriastrum procerum & $x$ & & & $\mathrm{x}$ & & ET \\
\hline & Sacaglotis procera & & & & & & \\
\hline \multirow[t]{7}{*}{ Lauraceae } & Aniba $s p$ & $x$ & $x$ & $x$ & & & \\
\hline & Aniba pertulis & & $x$ & $x$ & & & \\
\hline & Beilschmiedia rohliana & $x$ & & & & & \\
\hline & Endiicheria sp & & & $x$ & & & \\
\hline & Nectandra longifolia & & & $x$ & & & EP \\
\hline & Nectandra $s p$ & & & $\mathrm{x}$ & & & EP \\
\hline & Ocotea sernua & $\mathrm{x}$ & & & & & \\
\hline
\end{tabular}


Vegetación vascular y los complejos cenagosos. Z. Valoyes-Cardozo, G. Ramírez-Moreno

Tabla 1

Especies presentes en los ciénegas de la llanura aluvial del Atrato

\begin{tabular}{|c|c|c|c|c|c|c|c|}
\hline \multirow[t]{3}{*}{ Familia } & \multirow[t]{3}{*}{ Especie } & \multicolumn{6}{|c|}{ Complejos cenegosos } \\
\hline & & \multicolumn{2}{|c|}{ La Grande La Larga } & \multirow{2}{*}{$\begin{array}{l}\text { La Honda } \\
\text { Tanguí }\end{array}$} & \multirow{2}{*}{$\begin{array}{l}\text { Marriaga } \\
\text { Limón }\end{array}$} & \multirow{2}{*}{$\begin{array}{l}\text { Tumarado } \\
\text { Perancho }\end{array}$} & \multirow{2}{*}{$\begin{array}{l}\text { Gremio } \\
\text { Ecológico }\end{array}$} \\
\hline & & Beté & Tagachí & & & & \\
\hline & Ocotea sp & & & $x$ & & & \\
\hline \multirow[t]{15}{*}{ Lecythydaceae } & Cariniana pyriformis & $x$ & $x$ & & $x$ & & EP \\
\hline & Couropita guianensis & & $x$ & & & & EP \\
\hline & Couratari guianensis & & $x$ & & & & \\
\hline & Grias cauliflora & $x$ & & & & & \\
\hline & Grias sp & & & $x$ & & & \\
\hline & Gustavia gentry & $x$ & & & & & \\
\hline & Gustavia superba & $x$ & & $x$ & & & EP \\
\hline & Gustavia petiolata & $x$ & & & & & \\
\hline & Eschweilera integrifolia & & & $x$ & & & EP \\
\hline & Eschweilera pittierii & $x$ & & $x$ & & & \\
\hline & Eschweilera oligosperma & & $x$ & & & & \\
\hline & Eschweilera sp & & & $x$ & & & \\
\hline & Lecythis ampla & $x$ & & & & & EP \\
\hline & Lecythis tuyrana & $x$ & $x$ & & & & EP \\
\hline & Lecythis ollaria & & $x$ & & & & EP \\
\hline Loganiaceae & Strychnos jobertiana & $x$ & & & & & \\
\hline \multirow[t]{2}{*}{ Magnoliaceae } & Dugandiodendrom magnifolia & & $x$ & & & & \\
\hline & Dugandiodendron calophyllun & & $x$ & & & & \\
\hline \multirow[t]{3}{*}{ Malpigiaceae } & Bonisteriopsis martiniana vr $s$ & subenervia & $x$ & $x$ & & & \\
\hline & Bunchosia nítida & & & & $x$ & & \\
\hline & Malpiguia sp & & & $x$ & & & \\
\hline Malvaceae & Cavanillesia sp & & & $x$ & & & \\
\hline \multirow[t]{4}{*}{ Maranthaceae } & Calatea clotalifera & $x$ & & & & & \\
\hline & Calathea lutea & & $x$ & & $x$ & $x$ & \\
\hline & Calathea micans & & $x$ & & & & \\
\hline & Monotagma laxon & $x$ & & & & & \\
\hline \multirow[t]{3}{*}{ Margraviacae } & Souroubea guianensis & $x$ & $x$ & & & & \\
\hline & Margcgraviastrum sp & & $x$ & & & & \\
\hline & Pseudosarcopera sp & & & & $x$ & & \\
\hline \multirow[t]{5}{*}{ Meliaceae } & Carapa guianensis & $x$ & $x$ & & $x$ & & EP \\
\hline & Cedrela odorata & $x$ & $x$ & & $x$ & & $\mathrm{HD}$ \\
\hline & Guarea trichillioides & $x$ & & $x$ & & & EP \\
\hline & Guarea sp & & & $x$ & & & EP \\
\hline & Trichilia montana & & & & & $x$ & $H D$ \\
\hline \multicolumn{3}{|c|}{ Melastomataceae Acioti $s p$} & & $x$ & & & \\
\hline & Blakea sp & $x$ & & & & & \\
\hline & Clidemia Killipi & & & $x$ & & & \\
\hline
\end{tabular}


Bioetnia Volumen 9 № 2 (julio-diciembre), 2012

Tabla 1

Especies presentes en los ciénegas de la llanura aluvial del Atrato

\begin{tabular}{|c|c|c|c|c|c|c|c|}
\hline \multirow[t]{3}{*}{ Familia } & \multirow[t]{3}{*}{ Especie } & \multicolumn{6}{|c|}{ Complejos cenegosos } \\
\hline & & \multicolumn{2}{|c|}{ La Grande La Larga } & \multirow{2}{*}{$\begin{array}{c}\text { La Honda } \\
\text { Tanguí }\end{array}$} & \multirow{2}{*}{$\begin{array}{l}\text { Marriaga } \\
\text { Limón }\end{array}$} & \multirow{2}{*}{$\begin{array}{l}\text { Tumarado } \\
\text { Perancho }\end{array}$} & \multirow{2}{*}{$\begin{array}{l}\text { Gremio } \\
\text { Ecológico }\end{array}$} \\
\hline & & Beté & Tagachí & & & & \\
\hline & Monolena primuliflora & & & $x$ & & & \\
\hline & Blakea alternifolia & & $x$ & & & & \\
\hline & Blakea podagrica & & $x$ & & & & \\
\hline & Leandra granatensis & & $x$ & & & & \\
\hline & Miconia amplexans & $x$ & $x$ & & & & \\
\hline & Miconia argéntea & $x$ & $x$ & & & & \\
\hline & Miconia nervosa & & $x$ & & & & \\
\hline & Miconia pileata & $x$ & $x$ & $x$ & & & \\
\hline & Miconia rutencens & $x$ & $x$ & & & & \\
\hline & Miconia sp & & $x$ & & & & \\
\hline & Topobea parasítica & $x$ & $x$ & $x$ & & & \\
\hline & Topobea sp & $x$ & & & & & \\
\hline & Tococa guianensis & $x$ & & $x$ & & & \\
\hline Menispermaceae & Curarea sp & & & $x$ & & & \\
\hline \multirow[t]{10}{*}{ Mimosaceae } & Calliandra ssp & $x$ & & & & & \\
\hline & Inga acrocephala & & $x$ & & & & $\mathrm{HD}$ \\
\hline & Inga edulis & $x$ & & & & & $\mathrm{HD}$ \\
\hline & Inga chocoensis & $x$ & & & & & $\mathrm{HD}$ \\
\hline & Inga $s p$ & & $x$ & $x$ & & $x$ & $\mathrm{HD}$ \\
\hline & Parkia $s p$ & & & $x$ & & & ED \\
\hline & Pentaclethra macroloba & $x$ & $x$ & $x$ & $x$ & $x$ & EP \\
\hline & Pithecellobium jupumba & $x$ & & & & & \\
\hline & Pithecellobium longifolium & $x$ & & & & $x$ & \\
\hline & Zygia sp & $x$ & & & & & \\
\hline \multirow[t]{10}{*}{ Moraceae } & Artocarpus artilis & $x$ & & & & & \\
\hline & Brosimum guianensis & $x$ & & & & & ET \\
\hline & Brosimum utile & $x$ & $x$ & $x$ & & & EP \\
\hline & Clarisia sp & & & $x$ & & & \\
\hline & Ficus americana & $x$ & & & & & \\
\hline & Ficus glabrata & & $x$ & & & & EP \\
\hline & Ficus dendrocida & $\mathrm{x}$ & & & $x$ & & \\
\hline & Ficus insípida & & & & & $x$ & \\
\hline & Ficus sp & & & $x$ & & & \\
\hline & Pseudolmedia laevigata & $\mathrm{x}$ & & & & & \\
\hline \multirow[t]{4}{*}{ Myrtaceae } & Campomanesia lineatifolia & $\mathrm{x}$ & & & & & \\
\hline & Calycolpus calophyllus & & & $x$ & & & \\
\hline & Eugenia acapulcensis & & & & $x$ & $x$ & EP \\
\hline & Eugenia sp & $x$ & & $x$ & & & EP \\
\hline
\end{tabular}


Vegetación vascular y los complejos cenagosos. Z. Valoyes-Cardozo, G. Ramírez-Moreno

Tabla 1

Especies presentes en los ciénegas de la llanura aluvial del Atrato

\begin{tabular}{|c|c|c|c|c|c|c|c|}
\hline \multirow[t]{3}{*}{ Familia } & \multirow[t]{3}{*}{ Especie } & \multicolumn{6}{|c|}{ Complejos cenegosos } \\
\hline & & \multicolumn{2}{|c|}{ La Grande La Larga } & \multirow{2}{*}{$\begin{array}{l}\text { La Honda } \\
\text { Tanguí }\end{array}$} & \multirow{2}{*}{$\begin{array}{c}\text { Marriaga } \\
\text { Limón }\end{array}$} & \multirow{2}{*}{$\begin{array}{l}\text { Tumarado } \\
\text { Perancho }\end{array}$} & \multirow{2}{*}{$\begin{array}{l}\text { Gremio } \\
\text { Ecológico }\end{array}$} \\
\hline & & Beté & Tagachí & & & & \\
\hline \multirow[t]{10}{*}{ Myristicaceae } & Compsoneura otopa & & & & $x$ & & \\
\hline & Dialyanhtera gracilipes & $x$ & & & & $x$ & \\
\hline & Iryarthera ulei & $x$ & $x$ & & & & \\
\hline & Iryanthera tricornis & & & $x$ & & & \\
\hline & Iryanthera $s p$ & & & $x$ & & & \\
\hline & Otoba acuminata & & & $x$ & & & \\
\hline & Otoba lehemannii & & $x$ & & & & \\
\hline & Virola cuspidata & $x$ & & & & & EP \\
\hline & Virola flexuosa & $x$ & $x$ & & & & EP \\
\hline & Virola reedii & $x$ & & & & & EP \\
\hline Myrsinaceae & Ardisia manglillo & $x$ & $x$ & & & & \\
\hline \multirow[t]{3}{*}{ Ochnaceae } & Cespedecia spatulata & $x$ & & $x$ & & & $\mathrm{HD}$ \\
\hline & Cespedecia macrophilla & $x$ & & & & & $\mathrm{HD}$ \\
\hline & Sauvagesia erecta & & & $x$ & & & \\
\hline \multirow[t]{2}{*}{ Oleaceae } & Heisteria acuminata & $x$ & $x$ & $x$ & & & EP \\
\hline & Minquartia guianensis & & & $x$ & & & ET \\
\hline \multirow[t]{13}{*}{ Orchidaceae } & Erycina $s p$ & $x$ & & & & & \\
\hline & Dimerandra latipetala & $x$ & $x$ & & & & \\
\hline & Miltoniopsis roeslii & $x$ & & & & & \\
\hline & Psymorchys pusilla & $x$ & & & & & \\
\hline & Oncidium sp & $x$ & & $x$ & & & \\
\hline & Sobralia sp & $x$ & & & & & \\
\hline & Scaphyglotiis sp & & & $x$ & & & \\
\hline & Trigonidium argentinodium & $x$ & & & & & \\
\hline & Epidendrum nocturna & $x$ & $x$ & $x$ & & & \\
\hline & Epidendrum sp & $x$ & $x$ & $x$ & & & \\
\hline & Maxilaria $s p$ & $x$ & & $x$ & & & \\
\hline & Rudolphiela picta & $x$ & & & & & \\
\hline & Vainilla planifolia & & $x$ & & & & \\
\hline \multirow[t]{4}{*}{ Piperaceae } & Pereromia montium & $x$ & & & & & \\
\hline & Peperomia magnoliifolia & $x$ & & & & & \\
\hline & Piper arboreum & $x$ & & & & & \\
\hline & Piper sp & & & $x$ & & & \\
\hline Phyllanthaceae & Hieronyma sp & & & $x$ & & & \\
\hline \multirow[t]{4}{*}{ Poaceae } & Axonopus compressus & $x$ & & & $x$ & & \\
\hline & Gynerium sagittatum & & $x$ & $x$ & & & \\
\hline & Guadua angustifolia & $x$ & & & & & \\
\hline & Leersia hexandra & $x$ & & & $x$ & & \\
\hline
\end{tabular}


Bioetnia Volumen 9 № 2 (julio-diciembre), 2012

Tabla 1

Especies presentes en los ciénegas de la llanura aluvial del Atrato

\begin{tabular}{|c|c|c|c|c|c|c|c|}
\hline \multirow[t]{3}{*}{ Familia } & \multirow[t]{3}{*}{ Especie } & \multicolumn{6}{|c|}{ Complejos cenegosos } \\
\hline & & \multicolumn{2}{|c|}{ La Grande La Larga } & \multirow{2}{*}{$\begin{array}{l}\text { La Honda } \\
\text { Tanguí }\end{array}$} & \multirow{2}{*}{$\begin{array}{l}\text { Marriaga } \\
\text { Limón }\end{array}$} & \multirow{2}{*}{$\begin{array}{l}\text { Tumarado } \\
\text { Perancho }\end{array}$} & \multirow{2}{*}{$\begin{array}{l}\text { Gremio } \\
\text { Ecológico }\end{array}$} \\
\hline & & Beté & Tagachí & & & & \\
\hline & Pennisetum purpureum & & & & $x$ & & \\
\hline & Hymenachne amplexicaulis & $x$ & & $x$ & $x$ & & \\
\hline Rapataceae & Rapatea paludosa & & & $x$ & & & \\
\hline Rhizophoraceae & Rhizophora mangle & & & & $x$ & & \\
\hline \multirow[t]{18}{*}{ Rubiaceae } & Guattarda discolor & & $x$ & & & & \\
\hline & Genipa amaricana & $x$ & $x$ & & & $x$ & EP \\
\hline & Faramea multiflora & & & $x$ & & & \\
\hline & Faramea ocidentalis & & & $x$ & & & \\
\hline & Faramea sp & & & $x$ & & & \\
\hline & Hamelia sp & & $x$ & & & & \\
\hline & Psichotria poepigiana & $x$ & & & & & \\
\hline & Palicourea guianensis & $x$ & $x$ & & & & \\
\hline & Palicourea fastigiat & & & & $x$ & & \\
\hline & Cinchona sp & $x$ & $x$ & & & & \\
\hline & Condaminea corymbosa & & & $x$ & & & \\
\hline & Isertia sp & & & $x$ & & & \\
\hline & Pentagonia macrophylla & & & $x$ & & & \\
\hline & Posoqueria latifolia & & $x$ & & & & \\
\hline & Psychotria cinta & & & $x$ & & & \\
\hline & Psychotria poepigiana & & & $x$ & & & \\
\hline & Psychotria racemosa & & & $x$ & & & \\
\hline & Psychotria sp & & & $x$ & & & \\
\hline Rutaceae & Fagara sp & & & & & $x$ & \\
\hline Sapindaceae & Paulinia sp & & & $x$ & & & \\
\hline \multirow[t]{6}{*}{ Sapotaceae } & Chrysophyllum oleafolim & $x$ & & & & & ET \\
\hline & Chrysofilum superbum & & & $x$ & & & ET \\
\hline & Colocarpum sp & $x$ & & & & & \\
\hline & Manilkara sp & $x$ & & & & & \\
\hline & Pouteria caimito & & & $x$ & & & ET \\
\hline & Pouteris sp & $x$ & $x$ & $x$ & & & EP \\
\hline \multirow[t]{2}{*}{ Sterculiaceae } & Sterculia $s p$ & & & $x$ & & & \\
\hline & Sterculia apelata & & & & $x$ & & $\mathrm{HD}$ \\
\hline \multirow[t]{4}{*}{ Tiliaceae } & Apeiba aspera & $x$ & & & $x$ & & $\mathrm{HD}$ \\
\hline & Apeiba tibourbou & $x$ & & & & & $\mathrm{HD}$ \\
\hline & Apeiba membranacea & & $x$ & & & & \\
\hline & Luehea seemannii & $x$ & $x$ & & & $x$ & $\mathrm{HD}$ \\
\hline Urticaceae & Pourouma bicolor & & & $x$ & & & \\
\hline Vitaceae & Vitis tiliofila & $\mathrm{x}$ & & & & & \\
\hline
\end{tabular}


Tabla 1

Especies presentes en los ciénegas de la llanura aluvial del Atrato

\begin{tabular}{|c|c|c|c|c|c|c|c|}
\hline \multirow[t]{3}{*}{ Familia } & \multirow[t]{3}{*}{ Especie } & \multicolumn{6}{|c|}{ Complejos cenegosos } \\
\hline & & \multicolumn{2}{|c|}{ La Grande La Larga } & \multirow{2}{*}{$\begin{array}{c}\text { La Honda } \\
\text { Tanguí }\end{array}$} & \multirow{2}{*}{$\begin{array}{l}\text { Marriaga } \\
\text { Limón }\end{array}$} & \multirow{2}{*}{$\begin{array}{l}\text { Tumarado } \\
\text { Perancho }\end{array}$} & \multirow{2}{*}{$\begin{array}{l}\text { Gremio } \\
\text { Ecológico }\end{array}$} \\
\hline & & Beté & Tagachí & & & & \\
\hline \multirow[t]{2}{*}{ Vochysiaceae } & Vochysia jefensis & $x$ & & & & & $\mathrm{HD}$ \\
\hline & Vochysia ferruginea & $x$ & $x$ & $x$ & & & $\mathrm{HD}$ \\
\hline Zamiaceae & Zamia chigua & & & $x$ & & & \\
\hline
\end{tabular}

\section{Literatura citada}

Aldana, D, J. 2011. Transformación físico-espacial de los paisajes boscosos del sector sur del Parque Nacional Natural los Katíos (1989-2010). Perspect Geogr. 16: 31-54

BELFOR \& The Forest Management Trust-FMT. 2003. Consideraciones para árboles semilleros en bosques tropicales bajo manejo en Bolivia. Santa Cruz de la Sierra: Proyecto BOLFOR-The Forest Management Trust.

Cárdenas L., D., N. R. Salinas (eds.). 2007. Libro rojo de plantas de Colombia. Volumen 4. Especies maderables amenazadas: Primera parte. Serie libros rojos de especies amenazadas de Colombia. Bogotá: Instituto Amazónico de Investigaciones Científicas SINCHI-Ministerio de Ambiente, Vivienda y Desarrollo Territorial. $232 \mathrm{pp}$.

Carrasco, J., R. Flores. 2012. La palma africana especie exótica e invasora en los humedales costeros marinos de la vertiente Caribe de Honduras. I Congreso Iberoamericano de Gestión Integrada de Áreas Litorales, Cádiz del 25 al 27 de enero.

Castillo-Cortés, L. F., R. J. González. 2002. Evaluación de los humedales de los deltas de los rios San Juan y Baudó y ciénagas de Tumaradó, Perancho, La Honda y La Rica -bajo Atrato-departamento del Chocó, Sección Avifauna Acuática. Cali: Asociación para el Estudio y la Conservación de las Aves Acuáticas en Colombia. 42 p.

Corporación Nacional de Investigación y Fomento Forestal (CONIF y PIZANO SA) 1996. Manejo y conservación del ecosistema catival. Serie Técnica $N^{\circ}$ 44. Santa Fe de Bogotá: CONIF.

Condit, R., S.P. Hubbell, R.B. Foster. 1995. Demography and harvest potential of Latin American timber species: Data from a large, permanent plot in Panama. J Trop For Sci. 74: 599-622.

COMANA. 2006. Protección y manejo sustentable de humedales integrados a la cuenca hidrográfica. Volumen 2. Informe final. Santiago de Chile: COMANA.

CODESARROLLO. 2006. Implementación de 112 hectáreas en sistema agroforestal cacao-matarratón-maderable como medida de reactivación del sector y alternativa de producción para 56 pequeños productores en el municipio de Unguía-Chocó. Quidbó: Fundación Codesarrollo y Ministerio de Agricultura y Desarrollo Rural.

Corporación OSSO. 1998. Geología y geomorfología. URL disponible en: www.osso.org.co/docu/proyectos/grupo-osso/1998/atrato/geologia.pd

CORPOURABA. 2010. Humedales del río Atrato. URL disponible en: http:/ /www.corpouraba.gov.co/humedales-del-rio-atrato

Corporación para el Desarrollo Sostenible del Urabá (CORPOURABA), Corporación Autónoma Regional para el Desarrollo Sostenible del Chocó (CODECHOCO). 2006. Plan de manejo integrado de los humedales del Bajo y Medio Atrato. Quibdó: CORPOURABÁ; CODECHOCÓ. 540 p.

Corporación Autónoma Regional para el Desarrollo Sostenible del Chocó (CODECHOCÓ), Corporación para el Avance de la Región Pacífica y Darién Colombiano (CORPARIEN). 2011. Proyecto caracterización ecológica de las ciénagas de Marriaga y El Limón, municipio de Unguía, Chocó. Quibdó: CODECHOCÓ, CORPARIEN.

Cuesta, B. T., G. Ramírez M. 2009. Evaluación interdimensional de impactos ambientales sobre la dimensión física ocasionados por cultivos de palma aceitera y la ganadería extensiva en la selva tropical del Bajo Atrato. Rev Gestión y Ambiente. 12 (3): 37-48.

Chocó 7 Días. 2011. Queman cultivos en Curvaradó. Edición N 803, Quibdó, marzo 25 a 31 de 2011. URL disponible en http://www.choco7dias.com

El Tiempo. 1992. Incendio forestal en los bosques húmedos del Chocó. URL disponible en: http://www.eltiempo.com

Finegan. 1993. Curso intensivo internacional de silvicultura y manejo de bosques naturales tropicales. Mar. 1-Abr. 7: Turrialba, Costa Rica. I. Tema: bases ecológicas para la silvicultura. Turrialba. CATIE.

Galeano, G., R., Bernal, E. Calderón, N. García, A. Cogollo, A. Idárraga. 2005. Zamias. Pp. 387-436. En: Calderón, E., G. Galeano, N. García (eds). Libro Rojo de Plantas de Colombia. Volumen 2: Palmas, frailejones y zamias. Serie Libros rojos de especies amenazadas de Colombia. Bogotá:Instituto Alexander von Humboldt, Instituto de Ciencias Naturales de la Universidad Nacional de Colombia, Ministerio de Ambiente, Vivienda y Desarrollo Territorial.

Cogollo, A., C. Velásquez-Rúa, N. García. 2007. Las miristicáceas. Pp.155-92. En: García, N (ed.). Libro rojo de Plantas de Colombia. Volumen 5: Las magnoliáceas, las miristicáceas y las podocarpáceas. Serie Libros rojos de especies amenazadas de Colombia. Bogotá: Instituto Alexander von Humboldt, CORANTIOQUIA, Jardín Botánico Joaquín Antonio Uribe de Medellín, Instituto de Ciencias Naturales de la Universidad Nacional de Colombia, Ministerio de Ambiente, Vivienda y Desarrollo Territorial. $236 \mathrm{p}$.

González, H., H.D. Gómez, F. Arteaga. 1991. Aspectos estructurales de un bosque de cativo en la región del bajo Atrato, Colombia. Rev Fac Nal Agr. 44: $3-50$.

Gutiérrez, F. 2006. Estado de conocimiento de especies invasoras. Propuesta de lineamientos para el control de los impactos. Bogotá: Instituto de Investigación de Recursos Biológicos Alexander von Humboldt. 156 pp.

Herrera, C., J. A., J. L. Primera V. 2007. El cultivo de palma africana en las cuencas de los rios Curvaradó y Jiguamiandó, departamento del Chocó: desarrollo económico o exclusión social y cultural. Bucaramanga: Universidad Industrial de Santander.

Hidalgo, T. E. 2003. ¿Qué sabe usted sobre las ciénagas y la biodiversidad del Chocó? En: Chocó 7 días.

Hofstede, R., J. Lips, W. Jongsma, J. Sevink. 1998. Geografia, ecología y forestación de la Sierra Alta del Ecuador. Quito: Ediciones Abya-yala. $242 \mathrm{p}$.

Instituto de Investigaciones Ambientales del Pacífico (IIAP). 2012. Caracterización ecológica y sociocultural del complejo cenagoso La Larga, TagahíMedio Atrato, Chocó. Quibdó. Informe técnico. Quibdó: IIAP.

Instituto de Investigaciones Ambientales del Pacífico (IIAP). 2013. Caracterización ecológica y sociocultural de la Ciénega la Honda, TanguíMedio Atrato, Chocó. Quibdó. Informe técnico. Quibdó: IIAP.

Instituto Colombiano de Desarrollo Rural (INCODER). 2012. Caracterización jurídica y saneamiento de los territorios colectivos de Curvaradó y Jiguamiandó. Informe técnico elaborado por el INCODER, en cumplimiento de los autos 045 y 112 del 2012, proferidos por la Corte Constitucional, Bogotá, D.C., 12 de julio de 2012.

Instituto Nacional de Vías (INVIAS), Instituto de Investigaciones Ambientales 


\section{Bioetnia Volumen 9 № 2 (julio-diciembre), 2012}

del Pacífico (IIAP). 2013. Investigación para la complementación de los estudios de factibilidad (Fase II) para la navegabilidad del río Atrato. Análisis preliminar para el estudio de impacto ambiental. Informe final. Quibdó: INVIAS, IIAP.

Jaramillo-Villa, U., L. F. Jiménez-Segura. 2008. La pesca en las ciénagas de Tumarado, Bajo río Atrato, Colombia. Dahlia 10: 3-16.

Linares, R. 1998. Estudio preliminar de la asociación catival en Colombia: Bogotá: CONIF. $168 \mathrm{pp}$.

Marín M., G. A., M. C., Álvarez de U., J. Rosique G. 2004. Cultura alimentaria en el municipio de Acandí. Bol Antropol Universidad de Antioquia. 18 (35): 51-72.

Ministerio del Medio Ambiente. 2002. Política nacional para humedales interiores de Colombia. Bogotá: MinMedioAmbiente.

Ministerio del Medio Ambiente, CODECHOCO, IIAP y Municipio de Riosucio. 2002. Plan de ordenamiento territorial de las tierras colectivas de las comunidades negras del bajo Atrato: municipio de Riosucio-BajiraCarmen del Darién. Medellín: MinMedioAmbiente, CODECHOCO, IIAP y Municipio de Riosucio. $310 \mathrm{pp}$.

Mingorance, F., F. Minelli, H. Le Do. 2004. El cultivo de la palma africana en el Chocó, legalidad ambiental, territorial y derechos humanos. Quidbó: Diócesis de Quibdó y Human Rights Everywhere.

Olvera, V. 1988. Biología y ecología del lirio acuático Eichhornia crassipes (Mart.) Solms. Seminario-Taller Control y Aprovechamiento del Lirio Acuático, Cuernavaca, 18-20 enero de 1988.

Ramos P., Y. A. 2002. Evaluación de los humedales de los deltas de los ríos San Juan y Baudó y ciénagas de Tumarado, Perancho, la Honda y la Rica, bajo Atrato, departamento del Chocó. Caracterización botánica y zoológica. Quibdó: Instituto de Investigaciones del Pacífico (IIAP), Ministerio del Medio Ambiente de Colombia, WWF-Programa Colombia.
Rangel-Ch., J. O. 2004. Amenazas a la biota y a los ecosistemas del Chocó Biogeográfico. En: Rangel Ch, J. O. (ed.). Colombia Diversidad Biótica IV. El Chocó Biogeográfico/Costa Pacifica.

Ramírez, G., C. Z. Valoyes. 2009. Análisis de la vegetación acuática y terrestre del complejo cenagoso de la Grande de Beté, municipio del medio Atrato Chocó-Colombia- Bioetnia. 6 (1): 18-30.

Rodríguez, G, C. 2012. Determinación de rasgos funcionales del xilema en especies forestales tropicales de la cuenca media del río Atrato, Colombia. Trabajo de grado para optar al el título Ingeniero Forestal. Bogotá: Universidad Distrital Francisco José de Caldas, Facultad del Medio Ambiente y Recursos Naturales.

Sánchez, O. A. 2006. La ilegalidad del cultivo de palma en el Chocó. Agencia Prensa rural. UrL disponible en www.prensarural.org/spip/spip.php? article26

Ruiz, S, D. 2006. Nuevas formas de ser negro. Consideraciones sobre las identidades entre la gente chilapa y negra del Bajo Atrato. Colección Monografías, $\mathrm{N}^{\circ} 37$. Caracas: Programa Cultura, Comunicación y Transformaciones Sociales, CIPOST, FaCES, Universidad Central de Venezuela. 35 pp. URL disponible en: http://www.globalcult.org.ve/ monografias.htm

Unidad Administrativa Especial de Parques Nacionales Naturales de Colombia. Plan de Manejo 2007-2011. Parque Nacional Natural los Katios. Resumen ejecutivo. Medellín: UAESPNN, Dirección Territorial Noroccidente. 2007.

Yepes, A., J. del Valle, S. Jaramillo,S. A. Orrego. Recuperación estructural en bosques sucesionales andinos de Porce (Antioquia, Colombia). Rev Biol Trop [revista en la Internet]. 2010 Mar [citado 2014 Feb 25] ; 58 (1): 427445 